\title{
On $k$-Free Sequences of Integers
}

\author{
By Samuel S. Wagstaff, Jr.
}

\begin{abstract}
Let $A^{(k)}(n)$ denote the cardinality of the largest subsequence of $0,1,2, \cdots$, $n-1$, which contains no $k$ numbers in arithmetical progression. (Such a sequence is called $k$-free.) $A^{(k)}(n)$ is computed (on an IBM $360 / 65$ ) for $3 \leqq k \leqq 8$, and various values of $n$ to about 50. The results support the old conjecture that for all $k$, the limit $\tau^{(k)}=$ $\lim _{n \rightarrow \infty}\left(A^{(k)}(n)\right) / n=0$. The results $\tau^{(5)}<.649, \tau^{(6)}<.721, \tau^{(7)}<.776$, and $\tau^{(8)}<.8071$ are obtained. Several cases of a (disproved) conjecture of G. Szekeres are verified, including $A^{(5)}(94)=64$
\end{abstract}

An increasing sequence of positive integers is $k$-free $(k \geqq 2)$ if it does not have a subsequence of $k$ terms which forms an arithmetic progression. Let $A^{(k)}(n)$ denote the cardinality of the largest $k$-free subsequence of $0,1,2, \cdots, n-1$. Let $a^{(k)}(n)=$ $\left(A^{(k)}(n)\right) / n$. One can show that $\tau^{(k)}=\lim _{n \rightarrow \infty} a^{(k)}(n)$ exists. It is a well-known conjecture that $\tau^{(k)}=0$ for all $k$. K. F. Roth [5], [6] proved that $\tau^{(3)}=0$, and, recently, E. Szemerédi [9] showed that $\tau^{(4)}=0$. The values of the higher $\tau^{(k)}$ are not known, but F. Behrend [1] has proved that either all $\tau^{(k)}=0$ or $\lim _{k \rightarrow \infty} \tau^{(k)}=1$.

It is easy to see that $\tau^{(k)}=\inf _{n} a^{(k)}(n)$, so that it is of interest to compute $a^{(k)}(n)$ for particular $n$, since each value is an upper bound for $\tau^{(k)}$. P. Erdös and P. Turán [2] computed $A^{(3)}(n)$ for $1 \leqq n \leqq 21$. A. Makowski [3] and L. Moser [4] corrected and extended their work. In [10], I computed $A^{(4)}(n)$ for $1 \leqq n \leqq 52$ and $A^{(5)}(n)$ for $1 \leqq n \leqq 31$. Szemerédi's work has now superseded my results on $\tau^{(4)}$, and the present paper gives some values of $A^{(k)}(n)$ for $k=5,6,7$, and 8. The method used to compute these values was the same as that of [10], but since I used faster computers at Cornell University and the University of Rochester, I was able to go further. The results are shown in the table below. I carried the calculation further in the $A^{(5)}$ column because $\tau^{(5)}$ is the first unsettled case. That column represents about one hour of time on an IBM 360/65 computer. Each of the last three columns took about 15 minutes on the same computer.

The best results obtained were

$$
\begin{aligned}
& 0 \leqq \tau^{(5)} \leqq 48 / 74<.649, \\
& 0 \leqq \tau^{(6)} \leqq 31 / 43<.721, \\
& 0 \leqq \tau^{(7)} \leqq 38 / 49<.776, \text { and } \\
& 0 \leqq \tau^{(8)} \leqq 46 / 57<.8071 .
\end{aligned}
$$

These compare with $\tau^{(3)} \leqq 16 / 50=.32$ and $\tau^{(4)} \leqq 26 / 52=.5$, which were obtained in about the same amount of computer time.

Received August 30, 1971.

AMS 1970 subject classifications. Primary 10-04, 10L99; Secondary $10 \mathrm{~J} 99$.

Key words and phrases. $k$-free sequences, arithmetic progressions in sequences. 
SAMUEL S. WAGSTAFF, JR.

TABLE

\begin{tabular}{|c|c|c|c|c|c|c|}
\hline $\mathrm{n}$ & $A^{(3)}(n)$ & $A^{(4)}(n)$ & $A^{(5)}(n)$ & $A^{(6)}(n)$ & $A^{(7)}(n)$ & $A^{(8)}(n)$ \\
\hline 1 & 1 & 1 & 1 & 1 & 1 & 1 \\
\hline 2 & 2 & 2 & 2 & 2 & 2 & 2 \\
\hline 3 & 2 & 3 & 3 & 3 & 3 & 3 \\
\hline 4 & 3 & 3 & 4 & 4 & 4 & 4 \\
\hline 5 & 4 & 4 & 4 & 5 & 5 & 5 \\
\hline 6 & 4 & 5 & 5 & 5 & 6 & 6 \\
\hline 7 & 4 & 5 & 6 & 6 & 6 & 7 \\
\hline 8 & 4 & 6 & 7 & 7 & 7 & 7 \\
\hline 9 & 5 & 7 & 8 & 8 & 8 & 8 \\
\hline 10 & 5 & 8 & 8 & 9 & 9 & 9 \\
\hline 11 & 6 & 8 & 9 & 9 & 10 & 10 \\
\hline 12 & 6 & 8 & 10 & 10 & 11 & 11 \\
\hline 13 & 7 & 9 & 11 & 11 & 12 & 12 \\
\hline 14 & 8 & 9 & 12 & 12 & 12 & 13 \\
\hline 15 & 8 & 10 & 12 & 13 & 13 & 13 \\
\hline 16 & 8 & 10 & 13 & 13 & 14 & 14 \\
\hline 17 & 8 & 11 & 14 & 14 & 15 & 15 \\
\hline 18 & 8 & 11 & 15 & 15 & 16 & 16 \\
\hline 19 & 8 & 12 & 16 & 16 & 17 & 17 \\
\hline 20 & 9 & 12 & 16 & 17 & 18 & 18 \\
\hline 21 & 9 & 13 & 16 & 17 & 18 & 19 \\
\hline 22 & 9 & 13 & 16 & 18 & 19 & 19 \\
\hline 23 & 9 & 14 & 16 & 19 & 20 & 20 \\
\hline 24 & 10 & 14 & 17 & 20 & 21 & 21 \\
\hline 25 & 10 & 15 & 18 & 21 & 22 & 22 \\
\hline 26 & 11 & 15 & 18 & 22 & 23 & 23 \\
\hline 27 & 11 & 16 & 19 & 22 & 24 & 24 \\
\hline 28 & 11 & 17 & 20 & 22 & 24 & 25 \\
\hline 29 & 11 & 17 & 21 & 23 & 25 & 25 \\
\hline 30 & 12 & 18 & 21 & 23 & 26 & 26 \\
\hline
\end{tabular}


ON $k$-FREE SEQUENCES OF INTEGERS

\begin{tabular}{|c|c|c|c|c|c|c|}
\hline $\mathrm{n}$ & $A^{(3)}(n)$ & $A^{(4)}(n)$ & $A^{(5)}(n)$ & $A^{(6)}(n)$ & $A^{(7)}(n)$ & $A^{(8)}(n)$ \\
\hline 31 & 12 & 18 & 22 & 23 & 27 & 27 \\
\hline 32 & 13 & 18 & 22 & 24 & 28 & 28 \\
\hline 33 & 13 & 19 & 23 & 25 & 29 & 29 \\
\hline 34 & 13 & 20 & 24 & 25 & 30 & 30 \\
\hline 35 & 13 & 20 & 24 & 26 & 30 & 31 \\
\hline 36 & 14 & 20 & 25 & 27 & 31 & 31 \\
\hline 37 & 14 & 21 & 26 & 28 & 32 & 32 \\
\hline 38 & 14 & 21 & 27 & 28 & 33 & 33 \\
\hline 39 & 14 & 21 & 28 & 29 & 34 & 34 \\
\hline 40 & 15 & 22 & 28 & 30 & 35 & 35 \\
\hline 41 & 16 & 22 & 29 & 31 & 36 & 36 \\
\hline 42 & 16 & 22 & 30 & 31 & 36 & 37 \\
\hline 43 & 16 & 23 & 31 & 31 & 36 & 37 \\
\hline 44 & 16 & 23 & 32 & 32 & 36 & 38 \\
\hline 45 & 16 & 24 & 32 & 33 & 36 & 39 \\
\hline 46 & 16 & 24 & 32 & 34 & 37 & 40 \\
\hline 47 & 16 & 24 & 32 & 34 & 37 & 41 \\
\hline 48 & 16 & 25 & 32 & 35 & 38 & 42 \\
\hline 49 & 16 & 25 & 33 & 36 & 38 & 43 \\
\hline 50 & 16 & 26 & 33 & 37 & 39 & 44 \\
\hline 51 & 17 & 26 & 34 & 38 & 40 & 44 \\
\hline 52 & 17 & 26 & 35 & 38 & 41 & 44 \\
\hline 53 & 17 & & 35 & & 42 & 44 \\
\hline 54 & & & 36 & & & 45 \\
\hline 55 & & & 36 & & & 45 \\
\hline 56 & & & 37 & & & 46 \\
\hline 57 & & & 38 & & & 46 \\
\hline 58 & & & 39 & & & \\
\hline 59 & & & 40 & & & \\
\hline 60 & & & 40 & & & \\
\hline 61 & & & 41 & & & \\
\hline 62 & & & 42 & & & \\
\hline 63 & & & 43 & & & \\
\hline 64 & & & 44 & & & \\
\hline
\end{tabular}




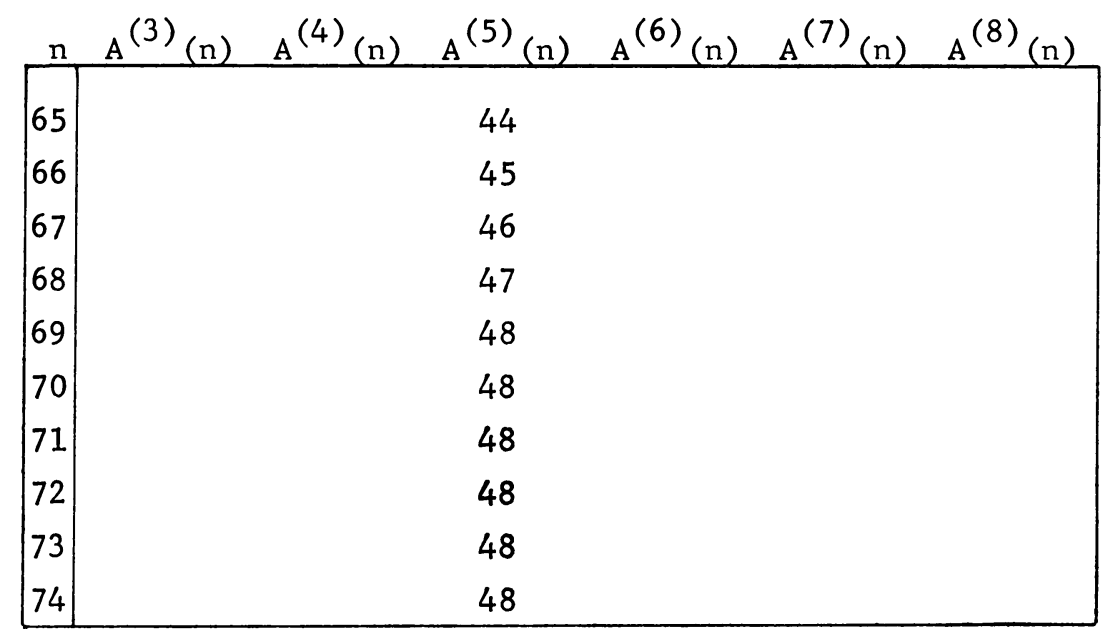

For prime* numbers $k$, it is well known that one can construct long $k$-free sequences as follows: Write down the first few integers to the base $k$ and delete those which contain the digit $k-1$. Equivalently, one can express the integers $0,1, \cdots, n-1$ to the base $k-1$ and interpret the representation in the base $k$. If $k$ is prime, then every arithmetic progression of length $k$ of integers written in the base $k$ must contain a number with the digit $k-1$. Since this does not happen in the sequence we constructed, it must be $k$-free. For example, with $k=5$, we get the 5 -free sequence

$$
0123567810111213151617182526 \cdots \text {. }
$$

This construction leads to the lower bound

$$
A^{(k)}\left(\frac{(k-2) k^{h}+1}{k-1}\right) \geqq(k-1)^{h}
$$

for primes $k$ and positive integers $h$. Equality always holds for $h=1$, and it also holds for the other values of $k$ and $h$ in the table, namely $A^{(3)}(5)=4, A^{(3)}(14)=8$, $A^{(3)}(41)=16, A^{(5)}(19)=16$, and $A^{(7)}(41)=36$.

G. Szekeres (see [2]) conjectured that equality held in all cases of (1) (for $k$ prime), but R. Salem and D. C. Spencer [7] disproved the conjecture by showing that

$$
A^{(3)}(n)>n^{1-c / \log \log n} \text {. }
$$

In [8], they prove that the conjecture fails for every $k$ by showing that $A^{(k)}(n)>c n^{1-\epsilon}$. I hoped to find the smallest counterexample to the conjecture, but the table could not be extended far enough. On the other hand, it is curious that there is enough information in the table to verify one more case of Szekeres' conjecture. For, using the obvious triangle inequality

$$
A^{(k)}(m+n) \leqq A^{(k)}(m)+A^{(k)}(n),
$$

and writing $A$ for $A^{(5)}$, we have

\footnotetext{
* For composite $k$, a similar construction works, but it is not nearly as efficient. See [8].
} 


$$
64=4^{3} \leqq A(94) \leqq A(74)+A(20)=48+16=64 .
$$

Thus, $A(94)=64$, which is another instance of the conjecture. A direct computer calculation of this result would certainly take many, many hours. Moreover,

$$
\begin{aligned}
64 & =A(94) \leqq A(95) \leqq A(96) \leqq A(97) \\
& \leqq A(74)+A(23)=48+16=64,
\end{aligned}
$$

so $A(94)=A(95)=A(96)=A(97)=64$.

But we can compute $A(n)$ for still more values of $n$ not in the table:

$$
\begin{aligned}
52 & =64-12=A(94)-A(15) \leqq A(79) \\
& \leqq A(74)+A(5)=48+4=52,
\end{aligned}
$$

so $A(79)=52$. Similarly, $A(84)=56$ and $A(89)=60$. Since $A(n+1)=A(n)$ or $A(n)+1$, we must have $A(75)=48$ or $49, A(76)=49$ or $50, \cdots, A(93)=63$ or 64 . The net result is that one would have to compute $A(n)$ up to $n$ larger than 100 to get much improvement in $\tau^{(5)} \leqq 48 / 74$. (Slight improvement is possible, e.g., if $A(75)=$ 48 or $A(76)=49$.)

My table and the above results for $A^{(5)}$ show that the $A^{(k)}$ functions grow about as slowly as could be hoped for in view of the work of Salem and Spencer, and I feel that they support the conjecture that all $\tau^{(k)}=0$. Of course, my evidence is very limited, but it seems impossible to extend the table much further with existing computers.

Department of Mathematics

University of Rochester

Rochester, New York 14627

1. F. BEHREND, "On sequences of integers containing no arithmetic progression," Casopis Mat. Fis. Praha, v. 67, 1938, pp. 235-239.

2. P. ERdös \& P. Turán, "On some sequences of integers," J. London Math. Soc. (2), v. 11,1936 , pp. $261-264$.

3. A. MakowsKI, "Remark on a paper of Erdös and Turán," J. London Math. Soc., v. 34, 1959, p. 480 . MR 21 \#5596.

4. L. Moser, "On non-averaging sets of integers," Canad. J. Math., v. 5, 1953, pp. 245252. MR 14, 726; 1278.

5. K. F. Rort, "On certain sets of integers," J. London Math. Soc., v. 28, 1953, pp. 104109. MR 14, 536; 1278 .

6. K. F. Roth, "Sur quelques ensembles d'entiers," C. R. Acad. Sci. Paris, v. 234, 1952, pp. 388-390. MR 13, 724 .

7. R. Salem \& D. C. SPencer, "On sets of integers which contain no three terms in arithmetic progression," Proc. Nat. Acad. Sci. U.S.A., v. 28, 1942, pp. 561-563. MR 4, 131.

8. R. Salem \& D. C. SPENCER, "On sets which do not contain a given number of terms in arithmetic progression," Nieuw Arch. Wisk. (2), v. 23, 1950, pp. 133-143. MR $11,417$.

9. E. SZEMERÉDI, "On sets of integers containing no four elements in arithmetic progression," Acta Math. Acad. Sci. Hungar., v. 20, 1969, pp. 89-104. MR 39 \#6861.

10. S. S. WAGSTAFF, "On sequences of integers with no 4 , or no 5 numbers in arithmetical progression," Math. Comp., v. 21, 1967, pp. 695-699. MR 36 \#5061. 